\title{
Unconditionally optimal convergence of an energy-conserving and linearly implicit scheme for nonlinear wave equations*
}

\author{
Waixiang $\mathrm{CaO}^{\dagger} \quad$ Dongfang $\mathrm{Li}^{\ddagger} \quad$ Zhimin Zhang§
}

\begin{abstract}
In this paper, we present and analyze an energy-conserving and linearly implicit scheme for solving the nonlinear wave equations. Optimal error estimates in time and superconvergent error estimates in space are established without time-step dependent on the spatial mesh size. The key is to estimate directly the solution bounds in the $H^{2}$-norm for both the nonlinear wave equation and the corresponding fully discrete scheme, while the previous investigations rely on the temporal-spatial error splitting approach. Numerical examples are presented to confirm energy-conserving properties, unconditional convergence, and optimal error estimates, respectively, of the proposed fully discrete schemes.
\end{abstract}

Keywords: scalar auxiliary variable, wave equations, stability, error estimate, superconvergence

AMS subject classifications: 65M60,65M12, 65N12

\section{Introduction}

We present an energy-conserving and linearly implicit scheme as well as the unconditionally optimal error estimates for solving the following wave equation

$$
\begin{array}{ll}
u_{t t}=\Delta u-\lambda u-F^{\prime}(u), & (\mathbf{x}, t) \in \Omega \times(0, T], \\
u(\mathbf{x}, 0)=u_{0}(\mathbf{x}), \quad u_{t}(\mathbf{x}, 0)=u_{1}(\mathbf{x}), & \mathbf{x} \in \Omega
\end{array}
$$

with the periodic boundary condition, where $\lambda \geq 0$ is a constant, $\Omega$ is a polygonal or polyhedral domain in $\mathbb{R}^{d}(d=2,3), u_{0}$ and $u_{1}$ are sufficiently smooth, and $F \in C^{2}(\mathbb{R})$ is the nonlinear potential. For simplicity, we assume that $\Omega$ is a rectangular or cubic domain. Nonlinear wave equations are widely used to model plenty of complicated natural phenomena in variety of scientific fields [3, 12, 13, 34. In the past several decades, it has been one of the hot spots in the numerical analysis of different schemes for the equations 4, 9, 16, 33, 35].

${ }^{*}$ This work is supported in part by the NSFC (Grant Nos. 11771128, 11871106, 11871092, 11926356) and NSAF (Grant No. U1930402).

${ }^{\dagger}$ School of Mathematical Sciences, Beijing Normal University, Beijing 100875, China (caowx@bnu.edu.cn)

${ }^{\ddagger}$ School of Mathematics and Statistics, Huazhong University of Science and Technology, Wuhan 430074, China (dfli@hust.edu.cn); and Hubei Key Laboratory of Engineering Modeling and Scientific Computing, Huazhong University of Science and Technology, Wuhan 430074, China

${ }^{\S}$ Beijing Computational Science Research Center, Beijing 100193, China (zmzhang@csrc.ac.cn); and Department of Mathematics, Wayne State University, Detroit, MI 48202, USA (zzhang@math.wayne.edu) 
There are many papers that consider error analysis of the fully discrete schemes for the nonlinear problems under the following assumption (e.g., [8, 10, 11, 17]),

$$
\left|F^{\prime}\left(u^{n}\right)-F^{\prime}\left(u_{h}^{n}\right)\right| \leq L\left|u^{n}-u_{h}^{n}\right|,
$$

where $u^{n}$ and $u_{h}^{n}$ are respectively the theoretical and numerical solutions, and $L>0$ is the Lipschitz coefficient. A classical model satisfying (1.2) is the Sine-Gorden equation, whose nonlinear term is $\sin (u)$. However, as pointed in [16], the assumption (1.2) is not the typical behavior of the general nonlinear wave equations and thus its range of the actual applicability is limited.

In order to deal with the non-Lipschitz nonlinearity, one common way is to impose a priori boundedness of the numerical approximations $u_{h}^{n}$. In classical finite element analysis, the numerical solutions in the maximum norm are usually estimated by

$$
\begin{aligned}
\left\|u_{h}^{n}\right\|_{L^{\infty}} & \leq\left\|R_{h} u^{n}\right\|_{L^{\infty}}+\left\|R_{h} u^{n}-u_{h}^{n}\right\|_{L^{\infty}} \\
& \leq\left\|R_{h} u^{n}\right\|_{L^{\infty}}+C h^{-d / 2}\left\|R_{h} u^{n}-u_{h}^{n}\right\|_{L^{2}} \\
& \leq\left\|R_{h} u^{n}\right\|_{L^{\infty}}+C h^{-d / 2}\left(\tau^{p}+h^{r+1}\right),
\end{aligned}
$$

where $R_{h}$ is the projection operator, $r+1$ and $p$ are convergence orders in spatial and temporal directions, respectively. Consequently, a time-step restriction $\tau=O\left(h^{\frac{d}{2 p}}\right)$ is needed in (1.3) (e.g., 7, 14, 15, 26, 27]). Such time-step restriction appears widely in the numerical analysis but is not always necessary in actual applications.

Unconditional convergence means that the established error bound is valid without the above mentioned time-step restriction. To achieve the unconditional convergence, a temporal-spatial error splitting approach is presented recently [19, 20, 21, 23, 24]. The key idea of the approach is to introduce a time discrete system, whose solution is denoted by $U^{n}$. Then, one can get the following error estimates

$$
\left\|U^{n}\right\|_{H^{2}} \leq C \quad \text { and } \quad\left\|R_{h} U^{n}-u_{h}^{n}\right\|_{L^{2}} \leq C h^{2} .
$$

The boundedness of the numerical solutions is obtained by

$$
\begin{aligned}
\left\|u_{h}^{n}\right\|_{L^{\infty}} & \leq\left\|R_{h} U^{n}-u_{h}^{n}\right\|_{L^{\infty}}+\left\|R_{h} U^{n}-\mathcal{I}_{h} U^{n}\right\|_{L^{\infty}}+\left\|\mathcal{I}_{h} U^{n}\right\|_{L^{\infty}} \\
& \leq C h^{-d / 2}\left(\left\|R_{h} U^{n}-u_{h}^{n}\right\|_{L^{2}}+\left\|R_{h} U^{n}-\mathcal{I}_{h} U^{n}\right\|_{L^{2}}\right)+\left\|U^{n}\right\|_{L^{\infty}} \\
& \leq C h^{2-d / 2}+\left\|U^{n}\right\|_{H^{2}} .
\end{aligned}
$$

Here $\mathcal{I}_{h} U^{n}$ denotes the interponation function of $U^{n}$. It implies the numerical solutions is bounded if the temporal and spatial step-sizes are sufficiently small, respectively. Then, the error estimates can be proved following the usual way. In spite of the interesting and instructive work, additional error estimates in different norms are required in the proof, and so far, most unconditional convergence results are focused on nonlinear parabolic problems.

Nonlinear wave equations (1.1) have several remarkable features. First, the models (1.1) are energy-conserving, i.e.,

$$
E(t)=\int_{\Omega} u_{t}^{2}+|\nabla u|^{2}+\lambda|u|^{2}+2 F(u) d x=E(0) .
$$

Second, the typical nonlinear terms are non-Lipschitz continuous. Third, the solutions have different regularities. A natural question is whether we can develop some effective 
unconditional convergence numerical schemes for nonlinear wave equations, taking all the remarkable features into account.

In the present paper, we present an energy-conserving and linearly implicit scheme for solving the nonlinear wave equations (1.1). The scheme is of order 2 in the temporal directions and no additional initial iterations are required. The construction of the scheme is based on recently-developed scalar auxiliary variable (SAV) approach combined with finite element methods, classical Crank-Nicolson methods, and extrapolation approximation. We show that our fully-discrete schemes conserve the energy and converge without any temporal step-size restrictions dependent on the spatial step-size. Unlike the previous temporal-spatial error splitting approach, we estimate the solution directly in the following procedure: (1) obtain the bounds in the $H^{2}$-norm of the solutions for both the nonlinear wave equations and the corresponding fully discrete schemes; (2) establish the bound for numerical solutions by applying the embedding inequality; (3) obtain the unconditionally optimal error estimates in time and superconvergent error estimates in space.

We remark that the key to construct the energy-conserving schemes is the SAV idea, which has been applied successfully to the gradient flows [1, 28, 29, 30]. Very recently, the idea was introduced to develop energy-conserving schemesfor the conservative laws [5, 6, 22, 25. However, much attention has been paid to the stability and energy-conserving properties, and no unconditional convergence results of fully discrete SAV schemes for nonlinear wave equations are found in the literature. This is the main motivation and contribution of the present study.

The rest of paper is organized as follows. In Section 2, we propose a fully discrete scheme for the nonlinear wave equation (1.1). In Section 3, we present a detailed proof to show the energy-conserving properties and unconditional convergence for the temporal discretization. Error estimates for the fully discrete solution is established in Section 4, where we prove that the approximation error is unconditionally optimal in time and superconvergent in space (under the $H^{1}$ norm). In Section 5, we present several numerical examples to confirm the theoretical results. Finally, conclusions are presented in Section 6.

\section{The linearly implicit method}

In this section, we present a fully discrete numerical scheme, which preserves the discrete energy.

Suppose $E_{1}(u)=\int_{\Omega} F(u) d \mathbf{x} \geq-c_{0}$ for some $c_{0}>0$, i.e., it is bounded from below, and let $C_{0}>c_{0}$ so that $E_{1}(u)+C_{0}>0$. We introduce the following scalar auxiliary variable $(\mathrm{SAV})$

$$
r(t)=\sqrt{E(u)}, \quad E(u)=\int_{\Omega} F(u) d \mathbf{x}+C_{0},
$$

and rewrite (1.1) as

$$
\begin{aligned}
& u_{t}=v, \\
& v_{t}=\triangle u-\lambda u-\frac{r}{\sqrt{E(u)}} f(u), \\
& r_{t}=\frac{1}{2 \sqrt{E(u)}} \int_{\Omega} f(u) u_{t} d \mathbf{x},
\end{aligned}
$$


where $f(u)=F^{\prime}(u)$.

Let $\mathcal{T}_{h}$ be the usual regular triangulation of the polygonal domain $\Omega$. Denote by $h_{T}$ the meshsize of $\mathcal{T}_{h}$, where $h_{T}$ is the diameter of the element $T \in \mathcal{T}_{h}$, and $h=\max _{T \in \mathcal{T}_{h}}$. Let $V_{h}$ be the classical finite-dimensional subspace of $H^{1}(\Omega)$, which consists of the usual continuous piecewise polynomials of degree $k(k \geq 1)$ on $\mathcal{T}_{h}$. That is,

$$
V_{h}=\left\{v \in C^{0}(\Omega):\left.v\right|_{T} \in \mathbb{P}_{k}, \forall T \in \mathcal{T}_{h}\right\},
$$

where $\mathbb{P}_{k}$ denotes the space of polynomials of degree no more than $k$.

Let $\tau=\frac{T}{N}$ with $N$ a given integer and $t_{n}=n \tau, n=0,1, \cdots, N$. Denote

$$
u^{n}=u\left(\mathbf{x}, t_{n}\right), \quad v^{n}=v\left(\mathbf{x}, t_{n}\right), \quad r^{n}=r\left(t_{n}\right) .
$$

For any sequence of the functions $\left\{f^{n}\right\}_{n=0}^{N}$, we define for all $n, n=0, \ldots N-1$

$$
D_{\tau} f^{n+1}:=\frac{D f^{n+1}}{\tau}=\frac{f^{n+1}-f^{n}}{\tau}, \quad \tilde{f}^{n+\frac{1}{2}}:=\frac{1}{2}\left(3 f^{n}-f^{n-1}\right), \quad \hat{f}^{n+\frac{1}{2}}:=\frac{f^{n+1}+f^{n}}{2} .
$$

Note that for $n=0$, we denote by $\tilde{f}^{\frac{1}{2}}=f^{0}$.

To design an energy-conserving and linearly implicit numerical scheme, which is easy to implement and efficient, we consider the following fully discrete Crank-Nicolson Galerkin SAV method: Find $u_{h}^{n+1} \in V_{h}, v_{h}^{n+1} \in V_{h}, r_{h}^{n+1} \in \mathbb{R}$ for $n=0, \ldots N-1$ such that for all $\left(w_{h}, \zeta_{h}\right) \in V_{h} \times V_{h}$

$$
\begin{aligned}
& \left(D_{\tau} u_{h}^{n+1}, w_{h}\right)=\left(\hat{v}_{h}^{n+\frac{1}{2}}, w_{h}\right), \\
& \left(D_{\tau} v_{h}^{n+1}, \zeta_{h}\right)=-\left(\nabla \hat{u}_{h}^{n+\frac{1}{2}}, \nabla \zeta_{h}\right)-\left(\lambda \hat{u}_{h}^{n+\frac{1}{2}}, \zeta_{h}\right)-\left(\frac{\hat{r}_{h}^{n+\frac{1}{2}}}{\sqrt{E\left(\tilde{u}_{h}^{n+\frac{1}{2}}\right)}} f\left(\tilde{u}_{h}^{n+\frac{1}{2}}\right), \zeta_{h}\right), \\
& r_{h}^{n+1}-r_{h}^{n}=\frac{1}{2 \sqrt{E\left(\tilde{u}_{h}^{n+\frac{1}{2}}\right)}} \int_{\Omega} f\left(\tilde{u}_{h}^{n+\frac{1}{2}}\right)\left(u_{h}^{n+1}-u_{h}^{n}\right) d \mathbf{x},
\end{aligned}
$$

where $(u, v)=\int_{\Omega} u(\mathbf{x}) v(\mathbf{x}) d \mathbf{x}, f\left(\tilde{u}_{h}^{\frac{1}{2}}\right)=f\left(u_{h}^{0}\right)$, and initial values are chosen as

$$
\left(u_{h}^{0}, v_{h}^{0}, r_{h}^{0}\right)=\left(R_{h} u_{0}, R_{h} u_{1}, \sqrt{E\left(u_{0}\right)}\right) .
$$

Here $R_{h} u_{0}$ is the Ritz projection of $u_{0}$, which will be defined later.

Equivalently, we rewrite the above scheme (2.2) into the following linear form

$$
\left(\left(4 I-\tau^{2} \triangle_{h}+\tau^{2} \lambda I\right) u_{h}^{n+1}, w_{h}\right)+\frac{\tau^{2}}{2}\left(u_{h}^{n+1}, b_{1}\right)\left(b_{1}, w_{h}\right)=\left(g, w_{h}\right)+\frac{\tau^{2}}{2}\left(u_{h}^{n}, b_{1}\right)\left(b_{1}, w_{h}\right)
$$

for all $w_{h} \in V_{h}$, where $\left(\triangle_{h} u_{h}, v_{h}\right):=-\left(\nabla u_{h}, \nabla v_{h}\right)$, and

$$
b_{1}=\frac{f\left(\tilde{u}_{h}^{n+\frac{1}{2}}\right)}{\sqrt{E\left(\tilde{u}_{h}^{n+\frac{1}{2}}\right)}}, \quad g=\left(4 I+\tau^{2} \triangle_{h}-\tau^{2} \lambda I\right) u_{h}^{n}+4 \tau v_{h}^{n}-2 \tau^{2} r_{h}^{n} b_{1} \text {. }
$$

Choosing $w_{h}$ in (2.3) to be the basis function of $V_{h}$ leads to a linear equation of the form

$$
A \mathbf{u}^{n+1}+\left(\mathbf{u}^{n+1}, \mathbf{b}_{1}\right) \mathbf{b}_{2}=\mathbf{g}
$$

for some matrix $A$ and vectors $\mathbf{b}_{1}, \mathbf{b}_{2}, \mathbf{g}$. By taking the inner product with $\mathbf{b}_{1}$ in the above equation, we obtain $\left(\mathbf{u}^{n+1}, \mathbf{b}_{1}\right)$ and then derive $\mathbf{u}^{n+1}$. Hence the scheme is easy to implement and very efficient. We also refer to [29, 30] for more detailed information. 


\section{Unconditionally energy preservation and convergence for the temporal discretization}

In this section, we shall prove that the Galerkin SAV method (2.2) preserves the energy unconditionally. Moreover, we establish the convergence analysis of the SAV approach with minimum assumptions.

We begin with the energy preservation property of the Galerkin SAV approach. We define the energy

$$
E^{n}=\sqrt{\frac{1}{2}\left(\left\|v_{h}^{n}\right\|^{2}+\left\|\nabla u_{h}^{n}\right\|^{2}+\lambda\left\|u_{h}^{n}\right\|^{2}\right)+\left(r_{h}^{n}\right)^{2}}, \quad 1 \leq n \leq N .
$$

Here $\|u\|^{2}=(u, u)=\|u\|_{L^{2}}^{2}$. Taking $\left(w_{h}, \zeta_{h}\right)=\left(v_{h}^{n+1}-v_{h}^{n}, u_{h}^{n+1}-u_{h}^{n}\right)$ and multiplying the third equation of (2.2) by $r_{h}^{n+1}+r_{h}^{n}$, we derive

$\frac{1}{2}\left(\left\|v_{h}^{n+1}\right\|^{2}-\left\|v_{h}^{n}\right\|^{2}+\left\|\nabla u_{h}^{n+1}\right\|^{2}-\left\|\nabla u_{h}^{n}\right\|^{2}+\lambda\left\|u_{h}^{n+1}\right\|^{2}-\lambda\left\|u_{h}^{n}\right\|^{2}\right)+\left(r_{h}^{n+1}\right)^{2}-\left(r_{h}^{n}\right)^{2}=0$.

Consequently,

$$
E^{n+1}=E^{n}=E^{0}, \quad \forall n \geq 1 .
$$

Now we consider a time-discrete system of equation:

$$
\begin{aligned}
& D_{\tau} U^{n+1}=\hat{V}^{n+\frac{1}{2}} \\
& D_{\tau} V^{n+1}=\triangle \hat{U}^{n+\frac{1}{2}}-\lambda \hat{U}^{n+\frac{1}{2}}-\frac{\hat{R}^{n+\frac{1}{2}}}{\sqrt{E\left(\tilde{U}^{n+\frac{1}{2}}\right)}} f\left(\tilde{U}^{n+\frac{1}{2}}\right), \\
& R^{n+1}-R^{n}=\frac{1}{2 \sqrt{E\left(\tilde{U}^{n+\frac{1}{2}}\right)}} \int_{\Omega} f\left(\tilde{U}^{n+\frac{1}{2}}\right)\left(U^{n+1}-U^{n}\right) d \mathbf{x},
\end{aligned}
$$

subject to the periodic boundary condition and the following initial conditions

$$
U^{0}(\mathbf{x})=u_{0}(\mathbf{x}), \quad V^{0}(\mathbf{x})=u_{1}(\mathbf{x}) .
$$

As we may observe, the numerical solution $\left(u_{h}^{n}, v_{h}^{n}, r_{h}^{n}\right)$ can be viewed as the Galerkin approximation of the above time-discrete system of equation. To study the convergence of the temporal discretization (3.1), we need some preliminaries.

First, for the simplicity of notations, throughout this paper, we denote by $C$ a generic positive constant, which depend solely upon the physical parameters of the problem and independent of $\tau, h, n$, and it is not necessary to be the same at every appearance. We adopt the usual notations for Sobolev spaces, e.g., $W^{m, p}(I)$ on sub-domain $I \in \Omega$ equipped with the norm $\|\cdot\|_{W^{m, p}, I}$ and semi-norm $|\cdot|_{W^{m, p}, I}$. We omit the index $I$ when $I=\Omega$. Especially, when $p=2$, we set $W^{m, p}(I)=H^{m}(I)$ and $\|\cdot\|_{W^{m, p}, I}=\|\cdot\|_{H^{m}, I}$ and $|\cdot|_{W^{m, p}, I}=|\cdot|_{H^{m}, I}$. The notation $\alpha \lesssim \beta$ implies that $\alpha$ is bounded by $\beta$ multiplied by a constant independent of $\tau, h, n$.

Second, we would like to present a Gronwall-type inequalities, which play important role in our later convergence analysis and error estimates. 
Lemma 1 ([18] ) Let $\tau, B$ and $a_{k}, b_{k}, c_{k}, \gamma_{k}$, for integers $k>0$, be nonnegative numbers such that

$$
a_{n}+\tau \sum_{k=0}^{n} b_{k} \leq \tau \sum_{k=0}^{n} \gamma_{k} a_{k}+\tau \sum_{k=0}^{n} c_{k}+B, \quad \text { for } n \geq 0 .
$$

Suppose that $\tau \gamma_{k}<1$, for all $k$, and set $\sigma_{k}=\left(1-\tau \gamma_{k}\right)^{-1}$. Then,

$$
a_{n}+\tau \sum_{k=0}^{n} b_{k} \leq\left(\tau \sum_{k=0}^{n} c_{k}+B\right) \exp \left(\tau \sum_{k=0}^{n} \gamma_{k} \sigma_{k}\right) .
$$

Lemma 2 ([2] ) Let $I=[a, b]$ and $\alpha(t), \beta(t), u(t) \in C^{0}(I)$. Suppose $\beta(t) \geq 0$ and

$$
u(t) \leq \alpha(t)+\int_{a}^{t} \beta(s) u(s) d s, \forall t \in I .
$$

Then

$$
u(t) \leq \alpha(t)+\int_{a}^{t} \alpha(s) \beta(s) e^{\int_{s}^{t} \beta(r) d r} d s, \quad \forall t \in I .
$$

Now we are ready to study the convergence of the solution of (3.1). Taking the inner product of the first two equations with $V^{n+1}-V^{n}, U^{n+1}-U^{n}$ and multiplying the third equation of (3.1) by $R^{n+1}+R^{n}$, we derive

$\frac{1}{2}\left(\left\|V^{n+1}\right\|^{2}-\left\|V^{n}\right\|^{2}+\left\|\nabla U^{n+1}\right\|^{2}-\left\|\nabla U^{n}\right\|^{2}+\lambda\left\|U^{n+1}\right\|^{2}-\lambda\left\|U^{n}\right\|^{2}\right)+\left(R^{n+1}\right)^{2}-\left(R^{n}\right)^{2}=0$,

which indicates that

$$
\left\|V^{n}\right\|+\left\|U^{n}\right\|_{H^{1}}+\left|R^{n}\right| \lesssim 1, \quad \forall 1 \leq n \leq N .
$$

As pointed out in [28, energy stable is not sufficient for the convergence which typically needs bounds in higher norms. Following the idea in [28], our convergence analysis is along this line: we first start from the energy preservation to derive the error bounds in higher norms, (i.e., the $H^{2}$ estimates) for the solution $U^{n}$, and thus get the $L^{\infty}$ for $U^{n}$ thanks to the embedding theory, and then we use the bounds in $H^{2}$ norms to show that the numerical solution $U^{n}$ converges to the exact solution $u^{n}$ in some suitable norms as $\tau$ tends to zero. To this end, we need to the bounds in $H^{2}$ norm of the PDE system (1.1). The error bounds for the solution of (3.1) is similar to those of the PDE system.

Note that most of the convergence and error analysis for linearly implicit are based on the so called Lipschitz assumption, i.e.,

$$
\left|F^{\prime}\left(u_{1}\right)-F^{\prime}\left(u_{2}\right)\right| \leq L\left|u_{1}-u_{2}\right|, \quad \forall u_{1}, u_{2} .
$$

The above assumption greatly limits its range of applicability. Following the basic idea of [28], we adopt the following assumption instead of the Lipschitz assumption in our convergence analysis:

$$
\begin{aligned}
& \left|f^{\prime}(x)\right|<C\left(|x|^{p}+1\right), \quad p \geq 0 \text { if } n=1,2 ; 0<p<4 \text { if } n=3, \\
& \left|f^{\prime \prime}(x)\right|<C\left(|x|^{p}+1\right), \quad p \geq 0 \text { if } n=1,2 ; 0<p<3 \text { if } n=3 .
\end{aligned}
$$


It has been proved in [28] that if $f(u)$ satisfies the conditions (3.3)-(3.4), there holds for some $\sigma$, where $0 \leq \sigma<1$, such that

$$
\left\|f^{\prime \prime}(u)\right\|_{L^{\infty}}+\left\|f^{\prime}(u)\right\|_{L^{\infty}} \leq C\left(1+\|\nabla \triangle u\|^{\sigma}\right) \leq \epsilon\|\nabla \triangle u\|+C_{\epsilon}
$$

for any $\epsilon>0$ with $C_{\epsilon}$ a constant depending on $\epsilon$.

We present the following estimates for the exact solution of (1.1).

Proposition 1 Assume that $u$ is the solution of (1.1), and $u_{0} \in H^{3}, u_{1} \in H^{2}$ and (3.3)(3.4) holds. Then for any $T>0$,

$$
\left(\|\triangle u\|+\left\|\triangle u_{t}\right\|+\|\nabla \triangle u\|\right)(T) \lesssim 1 .
$$

Proof. First, multiplying $u_{t}$ on both sides of (1.1) and using the integration by parts yields

$$
\frac{d}{d t}\left(\left\|u_{t}\right\|^{2}+\|\nabla u\|^{2}+\lambda\|u\|^{2}+2 \int_{\Omega} F(u)\right)=0,
$$

which indicates that

$$
\left\|u_{t}\right\|^{2}+\|u\|_{H^{1}}^{2}+\int_{\Omega} F(u) \lesssim 1
$$

On the other hand, we multiply $\triangle^{2} u_{t}$ on both sides of (1.1) and again use the integration by parts to obtain

$$
\frac{1}{2} \frac{d}{d t}\left(\left\|\triangle u_{t}\right\|^{2}+\|\nabla \triangle u\|^{2}+\lambda\|\triangle u\|^{2}\right)=-\left(\triangle f(u), \triangle u_{t}\right) .
$$

Integrating with respect to time between 0 to $t$ and using Cauchy-Schwarz inequality yields

$$
\begin{aligned}
\left(\left\|\triangle u_{t}\right\|^{2}+\|\nabla \triangle u\|^{2}+\lambda\|\Delta u\|^{2}\right)(t) & \leq\left(\left\|\triangle u_{t}\right\|^{2}+\|\nabla \triangle u\|^{2}+\lambda\|\Delta u\|^{2}\right)(0) \\
& +\int_{0}^{t}\left(\left\|\triangle u_{t}\right\|^{2}+\|\triangle f(u)\|^{2}\right)(t) d t
\end{aligned}
$$

By (3.5) and the identity

$$
\triangle f(u)=f^{\prime}(u) \triangle u+f^{\prime \prime}(u)|\nabla u|^{2},
$$

we have for all $0 \leq \delta<1$,

$$
\begin{aligned}
\|\triangle f(u)\| & \leq\left\|f^{\prime \prime}(u)\right\|_{L^{\infty}}\|\nabla u\|_{L^{4}}^{2}+\left\|f^{\prime}(u)\right\|_{L^{\infty}}\|\Delta u\| \\
& \leq C\left(\left\|f^{\prime \prime}(u)\right\|_{L^{\infty}}+\left\|f^{\prime}(u)\right\|_{L^{\infty}}\right)\left(\|\nabla u\|_{L^{4}}^{2}+\|\triangle u\|\right) \\
& \leq C\left(1+\|\nabla \triangle u\|^{\delta}\right)\left(\|\nabla u\|_{L^{4}}^{2}+\|\triangle u\|\right) .
\end{aligned}
$$

As for the term $\|\nabla u\|_{L^{4}}$, we use the Sobolev embedding theory and interpolation inequality about the spaces $H^{s}$ (see, e.g., 31]) and then obtain

$$
\|\nabla u\|_{L^{4}} \leq C\|\nabla u\|_{H^{d / 4}} \leq C\|\nabla u\|^{1-d / 8}\|\nabla \triangle u\|^{d / 8} \leq C\|\nabla \triangle u\|^{d / 8} .
$$

Moreover, by using the integration by parts and (3.6),

$$
\|\triangle u\|^{2} \leq C\|\nabla \triangle u\|\|\nabla u\| \leq C\|\nabla \triangle u\| .
$$


Consequently,

$$
\|\triangle f(u)\|^{2} \leq C\left(1+\|\nabla \triangle u\|^{2 \delta}\right)\left(\|\nabla u\|_{L^{4}}^{4}+\|\triangle u\|^{2}\right) \leq C\left(1+\|\nabla \triangle u\|^{2}\right) .
$$

Substituting the above inequality into (3.8) gives

$$
\begin{aligned}
\left(\left\|\triangle u_{t}\right\|^{2}+\|\nabla \triangle u\|^{2}+\lambda\|\triangle u\|^{2}\right)(t) & \lesssim\left(\left\|\triangle u_{t}\right\|^{2}+\|\nabla \triangle u\|^{2}+\lambda\|\triangle u\|^{2}\right)(0)+1+ \\
& +\int_{0}^{t}\left(\left\|\triangle u_{t}\right\|^{2}+\|\nabla \triangle u\|^{2}\right)(t) d t .
\end{aligned}
$$

By Gronwall inequality in Lemma 2, there holds

$$
\left(\left\|\triangle u_{t}\right\|^{2}+\|\nabla \triangle u\|^{2}+\lambda\|\triangle u\|^{2}\right)(t) \lesssim\left(\left\|\triangle u_{t}\right\|^{2}+\|\nabla \triangle u\|^{2}+\lambda\|\triangle u\|^{2}\right)(0)+1 \lesssim 1 .
$$

This finishes our proof.

Similar to the proof in the above Proposition, we also have the following $H^{2}$ estimates for the solution of (3.1).

Proposition 2 Assume that $\left(U^{n}, V^{n}, R^{n}\right)$ are the solutions of (3.1) and (3.3)-(3.4) holds. Then

$$
\left\|\triangle U^{n}\right\|+\left\|\triangle V^{n}\right\|^{2}+\left\|\nabla \triangle U^{n}\right\|^{2} \lesssim 1
$$

Proof. First, we have from the first equation of (3.1) that

$$
D_{\tau} \nabla \triangle U^{n+1}=\nabla \triangle \hat{V}^{n+\frac{1}{2}} .
$$

Multiplying the above equation with $\nabla\left(V^{n+1}-V^{n}\right)$ and the second equation of (3.1) with $\triangle^{2}\left(U^{n+1}-U^{n}\right)$, and then using the integration by parts, we obtain

$$
\begin{aligned}
& \left\|\nabla \triangle U^{n+1}\right\|^{2}-\left\|\nabla \triangle U^{n}\right\|^{2}+\left\|\triangle V^{n+1}\right\|^{2}-\left\|\triangle V^{n}\right\|^{2}+\lambda\left\|\triangle U^{n+1}\right\|^{2}-\lambda\left\|\triangle U^{n}\right\|^{2} \\
& =\frac{2 \hat{R}^{n+\frac{1}{2}}}{\sqrt{E\left(\tilde{U}^{n+\frac{1}{2}}\right)}}\left(\nabla f\left(\tilde{U}^{n+\frac{1}{2}}\right), \nabla \triangle\left(U^{n+1}-U^{n}\right)\right) .
\end{aligned}
$$

Denoting $f_{1}\left(\tilde{U}^{n+\frac{1}{2}}\right)=\frac{2 \hat{R}^{n+\frac{1}{2}} f\left(\tilde{U}^{n+\frac{1}{2}}\right)}{\sqrt{E\left(\tilde{U}^{n+\frac{1}{2}}\right)}}$ and summing up the above equation for all $n$ from 0 to $m$ yields

$$
\begin{aligned}
& \left\|\nabla \triangle U^{m+1}\right\|^{2}-\left\|\nabla \triangle U^{0}\right\|^{2}+\left\|\triangle V^{m+1}\right\|^{2}-\left\|\triangle V^{0}\right\|^{2}+\lambda\left\|\triangle U^{m+1}\right\|^{2}-\lambda\left\|\triangle U^{0}\right\|^{2} \\
& =\left(\nabla f_{1}\left(\tilde{U}^{m+\frac{1}{2}}\right), \nabla \triangle U^{m+1}\right)-\left(\nabla f_{1}\left(\tilde{U}^{\frac{1}{2}}\right), \nabla \triangle U^{0}\right)+\sum_{n=1}^{m}\left(\nabla\left(f_{1}\left(\tilde{U}^{n-\frac{1}{2}}\right)-f_{1}\left(\tilde{U}^{n+\frac{1}{2}}\right)\right), \nabla \triangle U^{n}\right) \\
& =\left(\nabla f_{1}\left(U^{m+1}\right), \nabla \triangle U^{m+1}\right)-\left(\nabla f_{1}\left(\tilde{U}^{\frac{1}{2}}\right), \nabla \triangle U^{0}\right)+I_{1}-I,
\end{aligned}
$$

where $f_{1}\left(U^{m+1}\right)=\frac{2 \hat{R}^{m+\frac{1}{2}} f\left(U^{m+1}\right)}{\sqrt{E\left(\tilde{U}^{m+\frac{1}{2}}\right)}}$, and

$$
I=\left(\nabla f_{1}\left(U^{m+1}\right)-\nabla f_{1}\left(\tilde{U}^{m+\frac{1}{2}}\right), \nabla \triangle U^{m+1}\right), \quad I_{1}=\sum_{n=1}^{m}\left(\nabla\left(f_{1}\left(\tilde{U}^{n-\frac{1}{2}}\right)-f_{1}\left(\tilde{U}^{n+\frac{1}{2}}\right)\right), \nabla \triangle U^{n}\right) .
$$


Since $R^{n}$ is bounded and $E(U)$ is bounded from below, we have from (3.5) that,

$$
\left\|\nabla f_{1}\left(U^{m+1}\right)\right\| \leq C\left(1+\left\|f^{\prime}\left(U^{m+1}\right)\right\|_{L^{\infty}}\right) \leq \epsilon\left\|\nabla \triangle U^{m+1}\right\|+C_{\epsilon} .
$$

Consequently,

$$
\left|\left(\nabla f_{1}\left(U^{m+1}\right), \nabla \triangle U^{m+1}\right)-\left(\nabla f_{1}\left(\tilde{U}^{\frac{1}{2}}\right), \nabla \triangle U^{0}\right)\right| \leq \frac{1}{4}\left\|\nabla \triangle U^{m+1}\right\|^{2}+\frac{1}{4}\left\|\nabla \triangle U^{0}\right\|^{2}+C .
$$

On the other hand, we note that

$$
\begin{aligned}
& \nabla f_{1}\left(\tilde{U}^{n-\frac{1}{2}}\right)-\nabla f_{1}\left(\tilde{U}^{n+\frac{1}{2}}\right) \\
& =f_{1}^{\prime}\left(\tilde{U}^{n-\frac{1}{2}}\right) \nabla \tilde{U}^{n-\frac{1}{2}}-f_{1}^{\prime}\left(\tilde{U}^{n+\frac{1}{2}}\right) \nabla \tilde{U}^{n+\frac{1}{2}} \\
& =f_{1}^{\prime}\left(\tilde{U}^{n-\frac{1}{2}}\right)\left(\nabla \tilde{U}^{n-\frac{1}{2}}-\nabla \tilde{U}^{n+\frac{1}{2}}\right)+\left(f_{1}^{\prime}\left(\tilde{U}^{n-\frac{1}{2}}\right)-f_{1}^{\prime}\left(\tilde{U}^{n+\frac{1}{2}}\right)\right) \nabla \tilde{U}^{n+\frac{1}{2}} \\
& =\frac{\tau}{2} f_{1}^{\prime}\left(\tilde{U}^{n-\frac{1}{2}}\right) \nabla\left(\hat{V}^{n-\frac{3}{2}}-3 \hat{V}^{n-\frac{1}{2}}\right)+\frac{\tau}{2} f_{1}^{\prime \prime}\left(\theta \tilde{U}^{n+\frac{1}{2}}+(1-\theta) \tilde{U}^{n-\frac{1}{2}}\right)\left(\hat{V}^{n-\frac{3}{2}}-3 \hat{V}^{n-\frac{1}{2}}\right) \nabla \tilde{U}^{n+\frac{1}{2}}
\end{aligned}
$$

for some $\theta \in(0,1)$, where in the last step, we have used the first equation of (3.1), which yields

$$
\nabla \tilde{U}^{n-\frac{1}{2}}-\nabla \tilde{U}^{n+\frac{1}{2}}=\frac{1}{2} \nabla\left(4 U^{n-1}-3 U^{n}-U^{n-2}\right)=\frac{\tau}{2} \nabla\left(\hat{V}^{n-\frac{3}{2}}-3 \hat{V}^{n-\frac{1}{2}}\right) .
$$

By (3.12) and the fact that

$$
\left\|V^{n}\right\|+\left\|\nabla U^{n}\right\| \lesssim 1
$$

we have

$$
\left\|\nabla f_{1}\left(\tilde{U}^{n-\frac{1}{2}}\right)-\nabla f_{1}\left(\tilde{U}^{n+\frac{1}{2}}\right)\right\|^{2} \leq \tau^{2}\left(\epsilon\left\|\nabla \triangle \tilde{U}^{n-\frac{1}{2}}\right\|^{2}+\epsilon\left\|\nabla \triangle \tilde{U}^{n+\frac{1}{2}}\right\|^{2}+\left\|\nabla\left(\hat{V}^{n-\frac{3}{2}}-3 \hat{V}^{n-\frac{1}{2}}\right)\right\|^{2}+C^{2}\right),
$$

and thus

$$
\left|I_{1}\right| \leq C \tau \sum_{n=1}^{m}\left(\left\|\nabla \triangle U^{n}\right\|^{2}+\left\|\nabla V^{n}\right\|^{2}\right)+C \tau .
$$

Similarly, there holds

$$
\left.\left\|\nabla f_{1}\left(\tilde{U}^{m+\frac{1}{2}}\right)-\nabla f_{1}\left(U^{m+1}\right)\right\|^{2} \leq \tau^{2} \epsilon \sum_{n=m-1}^{m+1}\left(\left\|\nabla V^{n}\right\|^{2}\right)+\left\|\nabla \triangle U^{n}\right\|^{2}\right)+C_{\epsilon} \tau^{2} .
$$

Then

$$
\left.|I| \leq \tau^{2} \sum_{n=m-1}^{m+1}\left(\left\|\nabla V^{n}\right\|^{2}\right)+\epsilon\left\|\nabla \triangle U^{n}\right\|^{2}\right)+C_{\epsilon} \tau^{2}+\frac{1}{4}\left\|\nabla \triangle U^{m+1}\right\|^{2} .
$$

Substituting (3.13), the estimates of $I_{1}$ and $I$ into (3.11) yields

$$
\begin{aligned}
\frac{1}{2}\left\|\nabla \triangle U^{m+1}\right\|^{2}+\left\|\triangle V^{m+1}\right\|^{2}+\lambda\left\|\triangle U^{m+1}\right\|^{2} & \leq\left\|\triangle V^{0}\right\|^{2}+\left\|\nabla \triangle U^{0}\right\|^{2}+\lambda\left\|\triangle U^{0}\right\|^{2} \\
& +C \tau \sum_{n=1}^{m+1}\left(\left\|\nabla \triangle U^{n}\right\|^{2}+\left\|\triangle V^{n}\right\|^{2}\right)+C .
\end{aligned}
$$

By Gronwall inequality, we have

$$
\left\|\nabla \triangle U^{m+1}\right\|^{2}+\left\|\triangle V^{m+1}\right\|^{2}+\lambda\left\|\triangle U^{m+1}\right\|^{2} \leq\left\|\triangle V^{0}\right\|^{2}+\left\|\nabla \triangle U^{0}\right\|^{2}+\lambda\left\|\triangle U^{0}\right\|^{2} \leq C .
$$


In case $\lambda=0$, we note that

$$
\triangle U^{m+1}=\triangle U^{m}+\frac{\tau}{2}\left(\triangle V^{m+1}+\triangle V^{m}\right)
$$

Then

$$
\left\|\triangle U^{m+1}\right\| \leq\left\|\triangle U^{m}\right\|+\frac{\tau}{2}\left\|\triangle V^{m+1}+\triangle V^{m}\right\|
$$

which yields

$$
\left\|\triangle U^{m+1}\right\| \leq\left\|\triangle U^{0}\right\|+C \leq C
$$

This finishes the proof of (3.10). The proof is complete.

As a direct consequence of (3.10) and the embedding inequality, we have

$$
\left\|U^{n}\right\|_{\infty} \leq C\left\|\triangle U^{n}\right\| \leq C, \quad \forall n .
$$

Remark 1 Following the same argument as that in [28], we conclude that: Assume that $u_{0} \in H^{3}$, when $\tau$ tends to zero, we have $U^{n} \rightarrow u^{n}$ strongly in $L^{\infty}\left(0, T ; H^{3-\epsilon}\right), \forall \epsilon>0$, weak-star in $L^{\infty}\left(0, T ; H^{3}\right), V^{n} \rightarrow v^{n}$ weak-star in $L^{\infty}\left(0, T ; H^{2}\right)$, and $R^{n} \rightarrow r^{n}$ weak-star in $L^{\infty}(0, T)$

Theorem 1 Suppose $u$ is the solution of (1.1), satisfying

$$
\left\|u_{0}\right\|_{H^{2}}+\|u\|_{L^{\infty}\left((0, T), H^{2}\right)}+\left\|u_{t}\right\|_{L^{2}\left((0, T), H^{2}\right)}+\left\|u_{t t}\right\|_{L^{2}\left((0, T), H^{2}\right)} \lesssim 1 .
$$

Then (3.1) admits a unique solution $\left(U^{n}, V^{n}, R^{n}\right)$ such that

$$
\left\|u^{n}-U^{n}\right\|_{H^{1}}+\left\|v^{n}-V^{n}\right\|+\left|r^{n}-R^{n}\right| \lesssim \tau^{2} .
$$

Proof. First we denote

$$
E_{u}^{n}=u^{n}-U^{n}, \quad E_{v}^{n}=v^{n}-V^{n}, \quad E_{r}^{n}=r^{n}-R^{n}, \quad H(u)=\frac{f(u)}{\sqrt{E(u)}} .
$$

By taking $t=t_{n+\frac{1}{2}}$ in (2.1) and using (3.1), we get

$$
\begin{aligned}
& D_{\tau} E_{u}^{n+1}=\hat{E}_{v}^{n+\frac{1}{2}}+T_{1}, \\
& D_{\tau} E_{v}^{n+1}=\triangle \hat{E}_{u}^{n+\frac{1}{2}}-\lambda \hat{E}_{u}^{n+\frac{1}{2}}-\hat{r}^{n+\frac{1}{2}} H\left(u^{n+\frac{1}{2}}\right)+\hat{R}^{n+\frac{1}{2}} H\left(\tilde{U}^{n+\frac{1}{2}}\right)+T_{2}, \\
& E_{r}^{n+1}-E_{r}^{n}=\frac{1}{2} \int_{\Omega} H\left(u^{n+\frac{1}{2}}\right)\left(u^{n+1}-u^{n}\right)-H\left(\tilde{U}^{n+\frac{1}{2}}\right)\left(U^{n+1}-U^{n}\right) d \mathbf{x}+T_{3},
\end{aligned}
$$

where $T_{i}$ denote the truncation errors, i.e.,

$$
\begin{aligned}
& T_{1}=D_{\tau} u^{n+1}-u_{t}^{n+\frac{1}{2}}+v^{n+\frac{1}{2}}-\hat{v}^{n+\frac{1}{2}} \\
& T_{2}=\triangle\left(u^{n+\frac{1}{2}}-\hat{u}^{n+\frac{1}{2}}\right)+D_{\tau} v^{n+1}-v_{t}^{n+\frac{1}{2}}-\lambda u^{n+\frac{1}{2}}+\lambda \hat{u}^{n+\frac{1}{2}}+\left(\hat{r}^{n+\frac{1}{2}}-r^{n+\frac{1}{2}}\right) H\left(u^{n+\frac{1}{2}}\right), \\
& T_{3}=\tau\left(D_{\tau} r^{n+1}-r_{t}^{n+\frac{1}{2}}\right)-\frac{\tau}{2} \int_{\Omega} H\left(u^{n+\frac{1}{2}}\right)\left(D_{\tau} u^{n+1}-u_{t}^{n+\frac{1}{2}}\right) d \mathbf{x} .
\end{aligned}
$$


Multiplying the first equation with $E_{v}^{n+1}-E_{v}^{n}$, the second equation with $E_{u}^{n+1}-E_{u}^{n}$, and the third equation with $E_{r}^{n+1}+E_{r}^{n}$ in (3.15), and then summing up three equalities, we get

$$
\begin{aligned}
& \frac{1}{2}\left(\left\|E_{v}^{n+1}\right\|^{2}-\left\|E_{v}^{n}\right\|^{2}+\left\|\nabla E_{u}^{n+1}\right\|^{2}-\left\|\nabla E_{u}^{n}\right\|^{2}+\lambda\left\|E_{u}^{n+1}\right\|^{2}-\lambda\left\|E_{u}^{n}\right\|^{2}\right)+\left|E_{r}^{n+1}\right|^{2}-\left|E_{r}^{n}\right|^{2} \\
& =\left(I_{2}, D E_{u}^{n+1}\right)+\left(T_{2}, D E_{u}^{n+1}\right)-\left(T_{1}, D E_{v}^{n+1}\right)+2\left(T_{3}+I_{3}\right) \hat{E}_{r}^{n+\frac{1}{2}}
\end{aligned}
$$

where

$$
\begin{aligned}
& I_{2}=\hat{r}^{n+\frac{1}{2}}\left(H\left(\tilde{U}^{n+\frac{1}{2}}\right)-H\left(u^{n+\frac{1}{2}}\right)\right), \\
& I_{3}=\frac{1}{2} \int_{\Omega}\left(H\left(u^{n+\frac{1}{2}}\right)-H\left(\tilde{U}^{n+\frac{1}{2}}\right)\right)\left(u^{n+1}-u^{n}\right) d \mathbf{x} .
\end{aligned}
$$

We next estimate the terms $T_{i}, I_{i}, i \leq 3$ respectively. By Taylor expansion, there holds

$$
\left\|T_{1}\right\| \lesssim \tau^{2}, \quad\left\|T_{2}\right\| \lesssim \tau^{2}, \quad\left|T_{3}\right| \lesssim \tau^{3}
$$

By (3.14) and the fact that $f \in C^{2}(\mathbb{R})$, we have

$$
\left|H\left(U^{n}\right)\right|+\left|H^{\prime}\left(U^{n}\right)\right|+\left|f^{\prime}\left(U^{n}\right)\right|+\left|f^{\prime \prime}\left(U^{n}\right)\right| \lesssim 1, \quad \forall n .
$$

Then there exists some $\theta \in(0,1)$ such that

$$
\begin{aligned}
\left\|H\left(\tilde{U}^{n+\frac{1}{2}}\right)-H\left(u^{n+\frac{1}{2}}\right)\right\| & =\left\|H^{\prime}\left(\theta \tilde{U}^{n+\frac{1}{2}}+(1-\theta) u^{n+\frac{1}{2}}\right)\left(\tilde{E}_{u}^{n+\frac{1}{2}}+\tilde{u}^{n+\frac{1}{2}}-u^{n+\frac{1}{2}}\right)\right\| \\
& \lesssim \tau^{2}+\left\|\tilde{E}_{u}^{n+\frac{1}{2}}\right\|,
\end{aligned}
$$

and thus

$$
\left\|I_{2}\right\| \lesssim \tau^{2}+\left\|\tilde{E}_{u}^{n+\frac{1}{2}}\right\|, \quad\left|I_{3}\right| \lesssim \tau\left(\tau^{2}+\left\|\tilde{E}_{u}^{n+\frac{1}{2}}\right\|\right)
$$

Consequently,

$$
\left|\left(T_{3}+I_{3}\right) \hat{E}_{r}^{n+\frac{1}{2}}\right| \lesssim \tau\left|\hat{E}_{r}^{n+\frac{1}{2}}\right|^{2}+\tau^{-1}\left(\left|I_{3}\right|^{2}+\left|T_{3}\right|^{2}\right) \lesssim \tau^{5}+\tau\left(\left|\hat{E}_{r}^{n+\frac{1}{2}}\right|^{2}+\left\|\tilde{E}_{u}^{n+\frac{1}{2}}\right\|^{2}\right) .
$$

Note that

$$
\left\|D E_{u}^{n+1}\right\|=\tau\left\|\hat{E}_{v}^{n+\frac{1}{2}}+T_{1}\right\| \lesssim \tau^{3}+\tau\left\|\hat{E}_{v}^{n+\frac{1}{2}}\right\|
$$

Then

$$
\left|\left(I_{2}+T_{2}, D E_{u}^{n+1}\right)\right| \lesssim \tau\left\|I_{2}\right\|^{2}+\tau\left\|T_{2}\right\|^{2}+\tau^{-1}\left\|D E_{u}^{n+1}\right\|^{2} \lesssim \tau^{5}+\tau\left(\left\|\hat{E}_{v}^{n+\frac{1}{2}}\right\|^{2}+\left\|\tilde{E}_{u}^{n+\frac{1}{2}}\right\|^{2}\right) .
$$

On the other hand, in light of the second equation of (3.15), we have

$$
\begin{aligned}
\left(T_{1}, D E_{v}^{n+1}\right) & =\tau\left(T_{1}, \triangle \hat{E}_{u}^{n+\frac{1}{2}}-\lambda \hat{E}_{u}^{n+\frac{1}{2}}-\hat{r}^{n+\frac{1}{2}} H\left(u^{n+\frac{1}{2}}\right)+\hat{R}^{n+\frac{1}{2}} H\left(\tilde{U}^{n+\frac{1}{2}}\right)+T_{2}\right) \\
& =-\tau\left(\nabla T_{1}, \nabla \hat{E}_{u}^{n+\frac{1}{2}}\right)+\tau\left(T_{1},-\lambda \hat{E}_{u}^{n+\frac{1}{2}}-\hat{r}^{n+\frac{1}{2}} H\left(u^{n+\frac{1}{2}}\right)+\hat{R}^{n+\frac{1}{2}} H\left(\tilde{U}^{n+\frac{1}{2}}\right)+T_{2}\right) .
\end{aligned}
$$

Noticing that

$$
\begin{aligned}
\left\|\hat{r}^{n+\frac{1}{2}} H\left(u^{n+\frac{1}{2}}\right)-\hat{R}^{n+\frac{1}{2}} H\left(\tilde{U}^{n+\frac{1}{2}}\right)\right\| & =\left\|\hat{E}_{r}^{n+\frac{1}{2}} H\left(u^{n+\frac{1}{2}}\right)+\hat{R}^{n+\frac{1}{2}}\left(H\left(u^{n+\frac{1}{2}}\right)-H\left(\tilde{U}^{n+\frac{1}{2}}\right)\right)\right\| \\
& \lesssim\left|E_{r}^{n+\frac{1}{2}}\right|+\tau^{2}+\left\|\tilde{E}_{u}^{n+\frac{1}{2}}\right\|,
\end{aligned}
$$


we have

$$
\left|\left(T_{1}, D E_{v}^{n+1}\right)\right| \lesssim \tau^{3}\left(\left\|\nabla \hat{E}_{u}^{n+\frac{1}{2}}\right\|+\left\|\hat{E}_{u}^{n+\frac{1}{2}}\right\|^{2}+\tau^{2}+\left|E_{r}^{n+\frac{1}{2}}\right|+\left\|\tilde{E}_{u}^{n+\frac{1}{2}}\right\|\right) .
$$

Plugging (3.19)-(3.21) into (3.16) yields

$$
\begin{aligned}
& \frac{1}{2}\left(\left\|E_{v}^{n+1}\right\|^{2}-\left\|E_{v}^{n}\right\|^{2}+\left\|\nabla E_{u}^{n+1}\right\|^{2}-\left\|\nabla E_{u}^{n}\right\|^{2}+\lambda\left\|E_{u}^{n+1}\right\|^{2}-\lambda\left\|E_{u}^{n}\right\|^{2}\right)+\left(E_{r}^{n+1}\right)^{2}-\left(E_{r}^{n}\right)^{2} \\
& \lesssim \tau^{5}+\tau\left(\left\|\tilde{E}_{u}^{n+\frac{1}{2}}\right\|^{2}+\left\|\hat{E}_{v}^{n+\frac{1}{2}}\right\|^{2}+\left\|\nabla \hat{E}_{u}^{n+\frac{1}{2}}\right\|^{2}+\left\|\hat{E}_{u}^{n+\frac{1}{2}}\right\|^{2}+\left|\hat{E}_{r}^{n+\frac{1}{2}}\right|^{2}\right) .
\end{aligned}
$$

Summing up all $n$ from 0 to $m$ and using the initial values, we get

$$
\begin{aligned}
& \left\|E_{v}^{m+1}\right\|^{2}+\left\|\nabla E_{u}^{m+1}\right\|^{2}+\lambda\left\|E_{u}^{m+1}\right\|^{2}+\left|E_{r}^{m+1}\right|^{2} \\
& \leq C \tau^{4}+C \tau \sum_{n=0}^{m+1}\left(\left\|E_{v}^{n}\right\|^{2}+\left\|\nabla E_{u}^{n}\right\|^{2}+\lambda\left\|E_{u}^{n}\right\|^{2}+\left|E_{r}^{n}\right|^{2}\right) .
\end{aligned}
$$

Then the desired result follows from the conclusion in Lemma 1 .

\section{Error estimates for the fully discrete solution}

In this section, we establish the error estimates for the solution of (2.2). By the error decomposition, we have

$$
w^{n}-w_{h}^{n}=w^{n}-W^{n}+W^{n}-w_{h}^{n}=w^{n}-W^{n}+e_{w}^{n}, \quad w=u, v, r .
$$

In light of the conclusion in Theorem 1, we only need to estimate the term $e_{w}, w=u, v, r$. To this end, we first define the Ritz projection operator $R_{h}: H_{0}^{1}(\Omega) \rightarrow V_{h}$ by

$$
\left(\nabla\left(v-R_{h} v\right), \nabla \omega\right)=0, \quad \forall \omega \in V_{h} .
$$

Then $e_{u}^{n}$ (similar for $e_{v}^{n}$ ) can be decomposed into

$$
e_{u}=U^{n}-u_{h}^{n}=\xi_{u}^{n}+\eta_{u}^{n}, \quad \xi_{u}^{n}=R_{h} U^{n}-u_{h}^{n}, \quad \eta_{u}=U^{n}-R_{h} U^{n} .
$$

According to the standard FEM theory [32], it holds that

$$
\left\|v-R_{h} v\right\|_{L^{2}}+h\left\|\nabla\left(v-R_{h} v\right)\right\|_{L^{2}} \leq C h^{s}\|v\|_{H^{s}}, \quad \forall v \in H^{s}(\Omega) \cap H_{0}^{1}(\Omega)
$$

for $1 \leq s \leq k+1$.

Note that the exact solutions of (3.1) satisfy

$$
\begin{aligned}
& \left(D_{\tau} U^{n+1}, w_{h}\right)=\left(\hat{V}^{n+\frac{1}{2}}, w_{h}\right), \\
& \left(D_{\tau} V^{n+1}, \zeta_{h}\right)=-\left(\nabla \hat{U}^{n+\frac{1}{2}}, \nabla \zeta_{h}\right)-\lambda\left(\hat{U}^{n+\frac{1}{2}}, \zeta_{h}\right)-\left(\hat{R}^{n+\frac{1}{2}} H\left(\tilde{U}^{n+\frac{1}{2}}\right), \zeta_{h}\right), \\
& R^{n+1}-R^{n}=\frac{1}{2} \int_{\Omega} H\left(\tilde{U}^{n+\frac{1}{2}}\right)\left(U^{n+1}-U^{n}\right) d \mathbf{x},
\end{aligned}
$$

Subtracting (2.2) from (4.2) gives the following error equation

$$
\begin{aligned}
& \left(D_{\tau} \xi_{u}^{n+1}, w_{h}\right)=\left(\hat{\xi}_{v}^{n+\frac{1}{2}}, w_{h}\right)+R_{1}\left(w_{h}\right), \\
& \left(D_{\tau} \xi_{v}^{n+1}, \zeta_{h}\right)=-\left(\nabla \hat{\xi}_{u}^{n+\frac{1}{2}}, \nabla \zeta_{h}\right)-\lambda\left(\hat{\xi}_{u}^{n+\frac{1}{2}}, \zeta_{h}\right)-\left(\hat{e}_{r}^{n+\frac{1}{2}} H\left(\tilde{u}_{h}^{n+\frac{1}{2}}\right), \zeta_{h}\right)+R_{2}\left(\zeta_{h}\right)+I_{2}\left(\zeta_{h}\right), \\
& e_{r}^{n+1}-e_{r}^{n}=\frac{1}{2} \int_{\Omega} H\left(\tilde{u}_{h}^{n+\frac{1}{2}}\right)\left(\xi_{u}^{n+1}-\xi_{u}^{n}\right) d \mathbf{x}+R_{3}+I_{3},
\end{aligned}
$$


where $H(u)=\frac{f(u)}{\sqrt{E(u)}}$, and

$$
\begin{array}{r}
R_{1}\left(w_{h}\right)=\left(\hat{\eta}_{v}^{n+\frac{1}{2}}, w_{h}\right)-\left(D_{\tau} \eta_{u}^{n+1}, w_{h}\right), \\
R_{2}\left(\zeta_{h}\right)=-\left(\nabla \hat{\eta}_{u}^{n+\frac{1}{2}}, \nabla \zeta_{h}\right)-\lambda\left(\hat{\eta}_{u}^{n+\frac{1}{2}}, \zeta_{h}\right)-\left(D_{\tau} \eta_{v}^{n+1}, \zeta_{h}\right), \\
I_{2}\left(\zeta_{h}\right)=\left(\hat{R}^{n+\frac{1}{2}}\left(H\left(\tilde{u}_{h}^{n+\frac{1}{2}}\right)-H\left(\tilde{U}^{n+\frac{1}{2}}\right)\right), \zeta_{h}\right), \\
R_{3}=\frac{1}{2} \int_{\Omega} H\left(\tilde{u}_{h}^{n+\frac{1}{2}}\right)\left(\eta_{u}^{n+1}-\eta_{u}^{n}\right) d \mathbf{x}, \\
I_{3}=\frac{1}{2} \int_{\Omega}\left(H\left(\tilde{U}^{n+\frac{1}{2}}\right)-H\left(\tilde{u}_{h}^{n+\frac{1}{2}}\right)\right)\left(U^{n+1}-U^{n}\right) d \mathbf{x} .
\end{array}
$$

Theorem 2 Suppose $u$ is the solution of (1.1) satisfying

$$
\left\|u_{0}\right\|_{H^{k+1}}+\|u\|_{L^{\infty}\left((0, T), H^{k+1}\right)}+\left\|u_{t}\right\|_{L^{2}\left((0, T), H^{k+1}\right)}+\left\|u_{t t}\right\|_{L^{2}\left((0, T), H^{k+1}\right)} \lesssim 1,
$$

and $\left(u_{h}^{n}, v_{h}^{n}, r_{h}^{n}\right)$ is the solution of (2.2) with $\left(u_{h}^{0}, v_{h}^{0}, r_{h}^{0}\right)=\left(R_{h} u_{0}, R_{h} u_{1}, \sqrt{E\left(u_{0}\right)}\right)$. Then

$$
\left\|R_{h} v^{n}-v_{h}^{n}\right\|+\left\|R_{h} u^{n}-u_{h}^{n}\right\|_{H^{1}}+\left|r^{n}-r_{h}^{n}\right| \lesssim h^{k+1}+\tau^{2} .
$$

Proof. We first estimate the terms $\xi_{u}^{n}, \xi_{v}^{n}$. By taking $\left(w_{h}, \zeta_{h}\right)=\left(D \xi_{v}^{n+1}, D \xi_{u}^{n+1}\right)$ and multiplying the third equation of (4.3) by $2 \hat{e}_{r}^{n+\frac{1}{2}}=e_{r}^{n+1}+e_{r}^{n}$, we derive

$$
\begin{aligned}
& \frac{1}{2}\left(\left\|\xi_{v}^{n+1}\right\|^{2}-\left\|\xi_{v}^{n}\right\|^{2}+\left\|\nabla \xi_{u}^{n+1}\right\|^{2}-\left\|\nabla \xi_{u}^{n}\right\|^{2}+\lambda\left\|\xi_{u}^{n+1}\right\|^{2}-\left\|\lambda \xi_{u}^{n}\right\|^{2}\right)+\left(e_{r}^{n+1}\right)^{2}-\left(e_{r}^{n}\right)^{2} \\
= & R_{2}\left(D \xi_{u}^{n+1}\right)+I_{2}\left(D \xi_{u}^{n+1}\right)-R_{1}\left(D \xi_{v}^{n+1}\right)+2\left(R_{3}+I_{3}\right) \hat{e}_{r}^{n+\frac{1}{2}}
\end{aligned}
$$

where $R_{i}, I_{i}, i \leq 3$ are given in (4.4). Summing up all $n$ from 0 to $m$ and using the initial error $\xi_{u}^{0}=\xi_{v}^{0}=0$, we get

$$
\begin{aligned}
& \frac{1}{2}\left(\left\|\xi_{v}^{m+1}\right\|^{2}+\left\|\nabla \xi_{u}^{m+1}\right\|^{2}+\lambda\left\|\xi_{u}^{m+1}\right\|^{2}\right)+\left|e_{r}^{m+1}\right|^{2} \\
& =\sum_{n=0}^{m}\left(R_{2}\left(D \xi_{u}^{n+1}\right)+I_{2}\left(D \xi_{u}^{n+1}\right)-R_{1}\left(D \xi_{v}^{n+1}\right)+2\left(R_{3}+I_{3}\right) \hat{e}_{r}^{n+\frac{1}{2}}\right) .
\end{aligned}
$$

To estimate the terms in the right hand side of (4.6), we shall first make the hypothesis that there exists a positive constant $C_{*}$ such that

$$
\left\|u_{h}^{n}\right\|_{L^{\infty}} \leq C_{*} .
$$

This hypothesis will be verified later by using the method of mathematical induction.

Due to (4.7) and the fact that $f \in C^{2}(\mathbb{R})$, we have

$$
\left|H\left(u_{h}^{n}\right)\right|+\left|H^{\prime}\left(u_{h}^{n}\right)\right|+\left|f^{\prime}\left(u_{h}^{n}\right)\right|+\left|f^{\prime \prime}\left(u_{h}^{n}\right)\right| \lesssim 1, \quad \forall n .
$$

Then

$$
\left|R_{3}\right| \lesssim \tau h^{k+1}, \quad\left|R_{2}\left(D \xi_{u}^{n+1}\right)\right| \lesssim h^{k+1}\left\|D \xi_{u}^{n+1}\right\| .
$$


By Taylor expansion, there exists a $\theta \in(0,1)$ such that

$$
\begin{aligned}
\left\|H\left(\tilde{u}_{h}^{n+\frac{1}{2}}\right)-H\left(\tilde{U}^{n+\frac{1}{2}}\right)\right\| & =\left\|H^{\prime}\left(\theta \tilde{u}_{h}^{n+\frac{1}{2}}+(1-\theta) \tilde{U}^{n+\frac{1}{2}}\right)\left(\tilde{u}_{h}^{n+\frac{1}{2}}-\tilde{U}^{n+\frac{1}{2}}\right)\right\| \\
& \lesssim h^{k+1}+\left\|\tilde{\xi}_{u}^{n+\frac{1}{2}}\right\| .
\end{aligned}
$$

Then

$$
\left|I_{2}\left(D \xi_{u}^{n+1}\right)\right| \lesssim\left(h^{k+1}+\left\|\tilde{\xi}_{u}^{n+\frac{1}{2}}\right\|\right)\left\|D \xi_{u}^{n+1}\right\|, \quad\left|I_{3}\right| \lesssim \tau\left(h^{k+1}+\left\|\tilde{\xi}_{u}^{n+\frac{1}{2}}\right\|\right),
$$

and thus

$$
\left|\sum_{n=0}^{m} 2\left(R_{3}+I_{3}\right) \hat{e}_{r}^{n+\frac{1}{2}}\right| \leq C h^{2(k+1)}+\tau \sum_{n=1}^{m}\left(\left\|\tilde{\xi}_{u}^{n+\frac{1}{2}}\right\|^{2}+\left|\hat{e}_{r}^{n+\frac{1}{2}}\right|^{2}\right) .
$$

On the other hand, we choose $w_{h}=D \xi_{u}^{n+1}$ in (4.3) to get

$$
\left\|D \xi_{u}^{n+1}\right\| \lesssim \tau\left(\left\|\hat{\xi}_{v}^{n+\frac{1}{2}}\right\|+h^{k+1}\right)
$$

which yields, together with Cauchy-Schwarz inequality

$$
\left|I_{2}\left(D \xi_{u}^{n+1}\right)\right|+\left|R_{2}\left(D \xi_{u}^{n+1}\right)\right| \lesssim \tau h^{2(k+1)}+\tau\left(\left\|\hat{\xi}_{v}^{n+\frac{1}{2}}\right\|^{2}+\left\|\tilde{\xi}_{u}^{n+\frac{1}{2}}\right\|^{2}\right) .
$$

Consequently,

$$
\sum_{n=0}^{m}\left|I_{2}\left(D \xi_{u}^{n+1}\right)\right|+\left|R_{2}\left(D \xi_{u}^{n+1}\right)\right| \lesssim h^{2(k+1)}+\tau \sum_{n=0}^{m}\left(\left\|\hat{\xi}_{v}^{n+\frac{1}{2}}\right\|^{2}+\left\|\tilde{\xi}_{u}^{n+\frac{1}{2}}\right\|^{2}\right) .
$$

As for the term $\sum_{n=0}^{m} R_{1}\left(D \xi_{v}^{n+1}\right)$ in (4.6), we recall the definition of $R_{1}$ in (4.4) to get

$$
\begin{aligned}
\left|\sum_{n=0}^{m} R_{1}\left(D \xi_{v}^{n+1}\right)\right| & =\left|\left(\hat{\eta}_{v}^{m+\frac{1}{2}}-D_{\tau} \eta_{u}^{m+1}, \xi_{v}^{m+1}\right)+\sum_{n=1}^{m}\left(\hat{\eta}_{v}^{n-\frac{1}{2}}-\hat{\eta}_{v}^{n+\frac{1}{2}}+D_{\tau} \eta_{u}^{n+1}-D_{\tau} \eta_{u}^{n}, \xi_{v}^{n}\right)\right| \\
& \leq C h^{k+1}\left\|\xi_{v}^{m+1}\right\|+C \tau h^{k+1} \sum_{n=1}^{m}\left\|\xi_{v}^{n}\right\| \\
& \leq C h^{2(k+1)}+C \tau \sum_{n=1}^{m}\left\|\xi_{v}^{n}\right\|^{2}+\frac{1}{4}\left\|\xi_{v}^{m+1}\right\|^{2} .
\end{aligned}
$$

Substituting the above inequality, (4.9)-(4.10) into (4.6), we get

$$
\left\|\xi_{v}^{m+1}\right\|^{2}+\left\|\nabla \xi_{u}^{m+1}\right\|^{2}+\left\|\xi_{u}^{m+1}\right\|^{2}+\left|e_{r}^{m+1}\right|^{2} \lesssim h^{2(k+1)}+\tau \sum_{n=0}^{m+1}\left(\left\|\xi_{v}^{n}\right\|^{2}+\left|e_{r}^{n}\right|^{2}+\left\|\xi_{u}^{n}\right\|^{2}\right) .
$$

By the Gronwall inequality given in Lemma 1,

$$
\left\|\xi_{v}^{n}\right\|+\left\|\xi_{u}^{n}\right\|_{H^{1}}+\left|e_{r}^{n}\right| \lesssim h^{k+1}, \quad \forall n \geq 1 .
$$

Then from the triangle inequality and the conclusion in Theorem 1,

$$
\begin{aligned}
& \left\|R_{h} v^{n}-v_{h}^{n}\right\|+\left\|R_{h} u^{n}-u_{h}^{n}\right\|_{H^{1}}+\left|r^{n}-r_{h}^{n}\right| \\
& \leq\left\|\xi_{v}^{n}\right\|+\left\|\xi_{u}^{n}\right\|_{H^{1}}+\left|e_{r}^{n}\right|+\left\|R_{h}\left(v^{n}-V^{n}\right)\right\|+\left\|R_{h}\left(u^{n}-U^{n}\right)\right\|+\left|r^{n}-R^{n}\right| \lesssim h^{k+1}+\tau^{2} .
\end{aligned}
$$

The proof is complete. 
Remark 2 Note that the optimal convergence rate for the $H^{1}$ error approximation is $O\left(h^{k}\right)$. The error estimate in (4.5) indicates that the Galerkin SAV solution $u_{h}^{n}$ is superclose to the Ritz projection of the exact solution $R_{h} u^{n}$ under the $H^{1}$ norm, which is one order higher than the counterpart optimal converge rate. As a direct consequence of (4.5), we have the following optimal error estimates:

$$
\begin{aligned}
& \left\|v^{n}-v_{h}^{n}\right\|_{H^{1}}+\left\|u^{n}-u_{h}^{n}\right\|_{H^{1}} \lesssim h^{k}+\tau^{2}, \\
& \left\|v^{n}-v_{h}^{n}\right\|+\left\|u^{n}-u_{h}^{n}\right\|+\left|r^{n}-r_{h}^{n}\right| \lesssim h^{k+1}+\tau^{2} .
\end{aligned}
$$

To end with this section, we prove the inequality (4.7).

Lemma 3 Under the conditions of Theorem 2, it holds that

$$
\left\|u_{h}^{n}\right\|_{L^{\infty}} \leq C_{*}, \quad \forall n \geq 1
$$

where the constant $C_{*}$ is independent of $\tau$ and $h$.

Proof. We will show the above inequality by induction. To this end, we first denote by $\mathcal{I}_{h} U \in V_{h}$ the interpolation function of $U$. By the approximation theory, we have

$$
\left\|\mathcal{I}_{h} U-U\right\|+\left\|\mathcal{I}_{h} U-R_{h} U\right\| \lesssim h^{2}\|U\|_{H^{2}}, \quad\left\|\mathcal{I}_{h} U\right\|_{L^{\infty}} \lesssim\|U\|_{L^{\infty}} .
$$

Note that

$$
\left\|u_{h}^{0}\right\|_{L^{\infty}}=\left\|R_{h} u^{0}\right\|_{L^{\infty}} \leq C .
$$

By (4.11) and the inverse inequality $\left\|v_{h}\right\|_{L^{\infty}} \lesssim h^{-\frac{d}{2}}\left\|v_{h}\right\|$ for all $v_{h} \in V_{h}$, we get

$$
\begin{aligned}
\left\|u_{h}^{1}\right\|_{L^{\infty}} & \leq\left\|u_{h}^{1}-R_{h} U^{1}\right\|_{L^{\infty}}+\left\|R_{h} U^{1}-\mathcal{I}_{h} U^{1}\right\|_{L^{\infty}}+\left\|\mathcal{I}_{h} U^{1}\right\|_{L^{\infty}} \\
& \leq C\left(h^{2-\frac{d}{2}}+\left\|U^{1}\right\|_{L^{\infty}}\right) \leq C_{1}\left(h^{2-\frac{d}{2}}+\left\|U^{1}\right\|_{H^{2}}\right) .
\end{aligned}
$$

Now we choose a positive constant $h_{1}$ which is small enough to satisfy

$$
C_{1} h_{1}^{\frac{1}{2}} \leq C
$$

Then for $h \in\left(0, h_{1}\right]$, we derive that

$$
\left\|u_{h}^{1}\right\|_{L^{\infty}} \leq C+\left\|U^{1}\right\|_{H^{2}} \leq C_{3} .
$$

Therefore, we can choose the positive constant $C_{*}$ independent of $h$ and $\tau$ such that

$$
C_{*} \geq \max \left\{2\left\|U^{n}\right\|_{H^{2}},\left\|u_{h}^{1}\right\|_{L^{\infty}}\right\} .
$$

Then (4.7) is valid for $n=1$. Next, suppose (4.7) holds for all $l \leq n-1$. We will show that it is also valid for $n$. Thanks to (4.11), we have

$$
\left\|u_{h}^{n}-R_{h} U^{n}\right\|_{H^{1}} \leq C h^{k+1} .
$$

Then

$$
\begin{aligned}
\left\|u_{h}^{n}\right\|_{L^{\infty}} & \leq\left\|u_{h}^{n}-R_{h} U^{n}\right\|_{L^{\infty}}+\left\|R_{h} U^{n}-\mathcal{I}_{h} U^{n}\right\|_{L^{\infty}}+\left\|\mathcal{I}_{h} U^{n}\right\|_{L^{\infty}} \\
& \leq C h^{-\frac{d}{2}}\left(\left\|u_{h}^{n}-R_{h} U^{n}\right\|+\left\|R_{h} U^{n}-\mathcal{I}_{h} U^{n}\right\|\right)+\left\|U^{n}\right\|_{L^{\infty}} \leq C_{1} h^{\frac{1}{2}}+\frac{C_{*}}{2} .
\end{aligned}
$$


Let $h_{1}$ be small enough to satisfy

$$
C_{1} h_{1}^{\frac{1}{2}} \leq \frac{C_{*}}{2}
$$

Then for $h \in\left(0, h_{1}\right]$, we derive that

$$
\left\|u_{h}^{n}\right\|_{L^{\infty}} \leq C_{1} h^{\frac{1}{2}}+\frac{C_{*}}{2} \leq C_{*}
$$

This completes the induction.

\section{$5 \quad$ Numerical simulations}

We present several numerical results to confirm our theoretical findings in this section.

Example 1 Consider the following Klein-Gordon equation

$$
u_{t t}=u_{x x}+u_{y y}+u-u^{3}+g_{1}(x, y, t) . \quad(x, y, t) \in[0,1]^{2} \times[0, T],
$$

where $u(x, y, 0), u_{t}(x, y, 0)$ and $g_{1}(x, y, t)$ are given by the exact solution

$$
u(x, t)=\exp (-t) x^{2}(1-x)^{2} y^{2}(1-y)^{2} .
$$

We test convergence orders of the fully discrete scheme using uniform triangulation with $M+1$ nodes in each spatial direction, and take $N=M$ and $N=M^{\frac{3}{2}}$ for the linear finite element method (L-FEM) and quadratic finite element method (Q-FEM), respectively. We list the errors at time $T=1$ as well as the convergence rates in Table 1. Here and below, we denote

$$
\|e\|_{0}=\left\|u^{N}-u_{h}^{N}\right\|, \quad\|e\|_{1}=\left\|R_{h} u^{N}-u_{h}^{N}\right\|_{H^{1}} .
$$

These results indicate that the fully discrete scheme is convergent and has the order $\mathcal{O}\left(\tau^{2}+\right.$ $\left.h^{r+1}\right)$. We also test the unconditional convergence of the fully discrete scheme with different spatial step-sizes for every fixed $\tau$. The $l_{2}$-errors at time $T=1$ are shown in Figure1. When the temporal stepsize is fixed, the $L_{2}$ errors tend to a constant. They imply that the error estimates hold without any temporal mesh sizes restrictions dependent on the spatial mesh sizes.
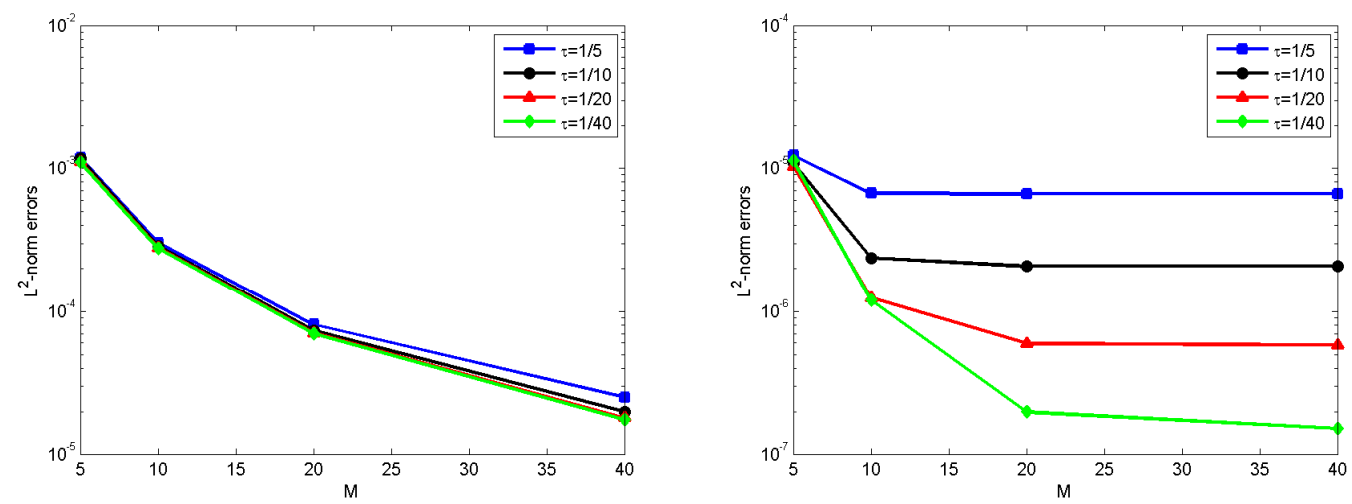

Figure 1: $L^{2}$-errors of linear and quadratic finite element approximation 
Table 1: Errors and convergent orders for 2D problems.

\begin{tabular}{ccccc|cccc}
\hline & \multicolumn{5}{c}{ L-FEM } & \multicolumn{4}{c}{ Q-FEM } \\
\cline { 2 - 9 } M & $\|e\|_{0}$ & order & $\|e\|_{1}$ & order & $\|e\|_{0}$ & order & $\|e\|_{1}$ & order \\
\hline 8 & $4.54 \mathrm{E}-4$ & - & $5.56 \mathrm{e}-4$ & - & $2.47 \mathrm{E}-6$ & - & $4.60 \mathrm{E}-5$ & - \\
16 & $1.11 \mathrm{E}-4$ & 2.03 & $1.61 \mathrm{E}-4$ & 1.83 & $2.71 \mathrm{E}-7$ & 3.19 & $6.85 \mathrm{E}-6$ & 2.74 \\
24 & $4.90 \mathrm{e}-5$ & 2.02 & $7.40 \mathrm{E}-5$ & 1.96 & $7.66 \mathrm{E}-8$ & 3.12 & $2.41 \mathrm{E}-6$ & 2.57 \\
32 & $2.74 \mathrm{e}-5$ & 2.02 & $4.24 \mathrm{E}-5$ & 1.96 & $3.18 \mathrm{E}-8$ & 3.05 & $9.81 \mathrm{E}-7$ & 3.10 \\
40 & $1.75 \mathrm{E}-5$ & 2.01 & $2.73 \mathrm{E}-5$ & 1.97 & $1.62 \mathrm{E}-8$ & 3.02 & $4.88 \mathrm{E}-7$ & 3.12 \\
\hline
\end{tabular}

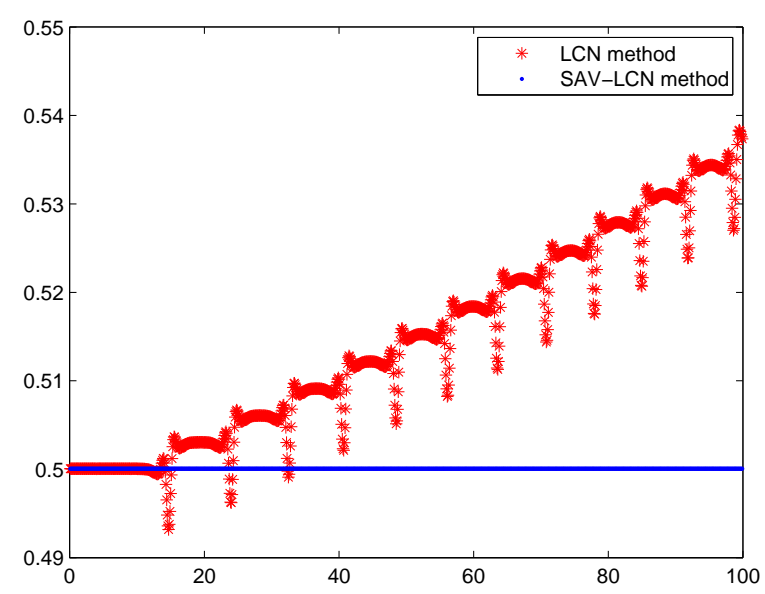

Figure 2: The evolutions of the discrete energies

Then, we set $g_{1}(x, y, t)=0, T=100, N=10, M=10$, and solve the problem using LFEM. The time discretization is achieved by the linearized Crank-Nicolson (LCN) method, and by the proposed SAV LCN method, respectively. The evolutions of the discrete energies are shown in Figure 2, Clearly, the energies obtained by the LCN finite element method increase as time goes on, while the one obtained by our method remains the same. It implies that numerical solutions by the SAV approach conserve the energy.

Example 2 Consider the following Sine-Gordon equation

$$
u_{t t}=u_{x x}+u_{y y}+u_{z z}+\sin (u)+g_{2}(x, y, z, t), \quad(x, y, z, t) \in[0,1]^{3} \times[0,1],
$$

where the initial conditions and $g_{2}(x, y, z, t)$ are produced from the exact solution

$$
u(x, t)=\left(1+t^{3}\right) \sin (2 \pi x) \sin (2 \pi y) \sin (2 \pi z) .
$$

We still take $N=M$ and $N=M^{\frac{3}{2}}$ for the linear and quadratic finite element approximations, respectively. The numerical errors at time $T=1$ as well as the convergence rates are presented in Table 2. The given results indicate that the fully discrete scheme has the order $\mathcal{O}\left(\tau^{2}+h^{r+1}\right)$.

Next, we set $g_{2}(x, y, z, t)=0, T=20, N=10, M=10$ and solve the problem by the linear finite element method. The evolutions of the discrete energies for the 3D problems are displayed in Fig. 3. Clearly, the discrete energies by the SAV approach remain unchanged, 
Table 2: Errors and convergent orders for 3D problems.

\begin{tabular}{ccccc|ccccc}
\hline & \multicolumn{4}{c|}{ L-FEM } & & \multicolumn{4}{c}{ Q-FEM } \\
\cline { 2 - 7 } \cline { 7 - 9 } M & $\|e\|_{0}$ & order & $\|e\|_{1}$ & order & & $\|e\|_{0}$ & order & $\|e\|_{1}$ & order \\
\hline 16 & $6.36 \mathrm{E}-2$ & - & $4.43 \mathrm{E}-1$ & - & 10 & $5.69 \mathrm{E}-3$ & - & $1.29 \mathrm{E}-1$ & - \\
20 & $4.17 \mathrm{E}-2$ & 1.89 & $2.94 \mathrm{E}-1$ & 1.83 & 12 & $3.24 \mathrm{E}-3$ & 3.09 & $7.41 \mathrm{E}-2$ & 2.96 \\
24 & $2.94 \mathrm{E}-2$ & 1.92 & $2.08 \mathrm{E}-1$ & 1.91 & 14 & $1.99 \mathrm{E}-3$ & 3.16 & $4.91 \mathrm{E}-2$ & 2.67 \\
28 & $2.18 \mathrm{E}-2$ & 1.94 & $1.55 \mathrm{E}-1$ & 1.90 & 16 & $1.31 \mathrm{E}-3$ & 3.13 & $3.40 \mathrm{E}-2$ & 2.75 \\
\hline
\end{tabular}

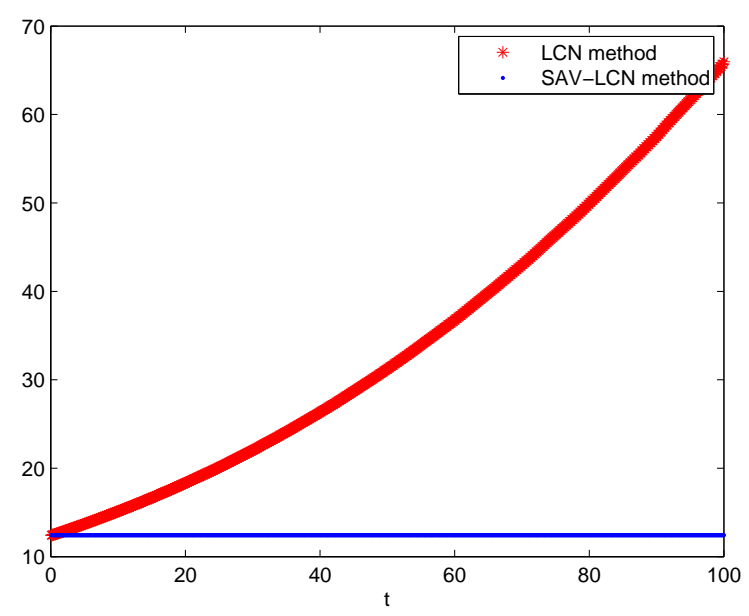

Figure 3: The evolutions of the discrete energies

while the ones obtained by the LCN finite element method increase as time goes on. They further confirm the findings in this study.

\section{Conclusion}

In this study, we present a linearly implicit numerical schemes for solving the nonlinear wave equation (1.1). The scheme is developed by combining the SAV approach with finite element methods, classical Crank-Nicolson methods, and extrapolation approximation. The fully discrete scheme is proved to be unconditionally convergent and energy-conserving. Numerical illustrations are presented to confirm the theoretical findings.

\section{References}

[1] G. Akrivis, B. Li, and D. Li, Energy-Decaying Extrapolated RK-SAV Methods for the Allen-Cahn and Cahn-Hilliard Equations, SIAM. J. Sci. Comput., 41 (2019) A3703-A3727.

[2] R. Bellman, The stability of solutions of linear differential equations, Duke Math. J., 10 (1943), 643-647.

[3] A. Biswas, Soliton perturbation theory for phi-four model and nonlinear Klein-Gordon equations., Commun. Nonlinear Sci. Numer. Simul., 14 (2009) 3239-3249. 
[4] L. Brugnano, G. F. Caccia, and F. Iavernaro, Energy conservation issues in the numerical solution of the semilinear wave equation, Appl. Math. Comput., 270 (2015) 842-870.

[5] W. Cai, C. Jiang, Y. Wang, and Y. Song, Structure-preserving algorithms for the twodimensional sine-Gordon equation with Neumann boundary conditions, J. Comput. Phys., 395 (2019) 166-185.

[6] J. Cai and J. Shen, Two classes of linearly implicit local energy-preserving approach for general multi-symplectic Hamiltonian PDEs, J. Comput. Phys., (2019) https://doi.org/10.1016/j.jcp.2019.108975.

[7] J. R. Cannon and Y. Lin, Nonclassical H1 projection and Galerkin methods for nonlinear parabolic integro-differential equations, SIAM. J. Numer. Anal., 25 (1988) 187201.

[8] B. Cano and M. J. Moreta, Multistep cosine methods for second-order partial differential systems, IMA J. Numer. Anal., 30 (2010) 431-461.

[9] W. Cao, D. Li, and Z. Zhang, Optimal superconvergence of energy conserving local discontinuous Galerkin methods for wave equations, Commu. Comput. Phys., 21 (2017) 211-236.

[10] C. Carstensen and G. Dolzmann, Time-Space Discretization of the Nonlinear Hyperbolic System $u_{t t}=\operatorname{div}\left(\sigma(\mathbf{D} U)+\mathbf{D} U_{t}\right)$, SIAM. J. Numer. Anal., 42 (2004) 75-89.

[11] J. E. Dendy, Galerkin's Method for Some Highly Nonlinear Problems, SIAM. J. Numer. Anal., 14 (1977) 327-347.

[12] R. K. Dodd, I. C. Eilbeck, J. D. Gibbon, and H. C. Morris, Solitons and Nonlinear Wave Equations., Academic, 1982.

[13] P. J. Drazin and R. S. Johnson, Solitons: An Introduction, Phy. Tod., 43 (1990) 70-71.

[14] I. Farago, Finite element method for solving nonlinear parabolic equations, Computers Math. Applic., 21 (1991) 59-69.

[15] S. Garcia, Improved error estimates for mixed finite-element approximations for nonlinear parabolic equations: the discrete-time case, Numer. Methods Partial Differ. Equ., 10 (1994) 149-169.

[16] L. Gauckler, Error analysis of trigonometric integrators for semilinear wave equations, SIAM J. Numer. Anal., 53 (2015) 1082-1106.

[17] V. Grimm, A note on the Gautschi-type method for oscillatory second-order differential equations, Numer. Math., 102 (2005) 61-66.

[18] J. G. Heywood and R. Rannacher, Finite element approximation of the nonstationary Navier-Stokes problem IV: Error analysis for second-order time discretization, SIAM. J. Numer. Anal., 27 (1990) 353-384. 
[19] B. Li, H. Gao, and W. Sun, Unconditional optimal error estimates of a Crank-Nicolson Galerkin method the nonlinear thermistor equations, SIAM J. Numer. Anal., 52 (2014) 933-954.

[20] B. Li and W. Sun, Error analysis of linearized semi-implicit Galerkin finite element methods for nonlinear parabolic equations, Int. J. Numer. Anal. Model., 10 (2013) 622-633.

[21] B. Li and W. Sun, Unconditional convergence and optimal error estimates of a Galerkin-mixed FEM for incompressible miscible flow in porous media, SIAM J. Numer. Anal., 51 (2013) 1959-1977.

[22] D. Li and W. Sun, Linearly Implicit and High-Order Energy-Conserving Schemes for Nonlinear Wave Equations, J. Sci. Comput., 83 (2020) 65.

[23] D. Li, J. Wang, and J. Zhang, Unconditionally convergent L1-Galerkin FEMs for nonlinear time-fractional Schrödinger equations, SIAM. J. Sci. Comput., 39 (2017) A3067-A3088.

[24] D. Li, C. Wu, and Z. Zhang, Linearized Galerkin FEMs for Nonlinear Time Fractional Parabolic Problems with Non-smooth Solutions in Time Direction, J. Sci. Comput., 80 (2019) 403-419.

[25] X. Li, J. Wen, D. Li, Mass- and energy-conserving difference schemes for nonlinear fractional Schrödinger equations. Appl Math Lett, 2021, 111: 106686.

[26] M. Luskin, A Galerkin method for nonlinear parabolic equations with nonlinear boundary conditions, SIAM J. Numer. Anal. 16 (1979) 284-299.

[27] H. H. Rachford Jr., Two-level discrete-time Galerkin approximations for second order nonlinear parabolic partial differential equations, SIAM J. Numer. Anal., 10 (1973) $1010-1026$.

[28] J. Shen and J. Xu, Convergence and Error Analysis for the Scalar Auxiliary Variable (SAV) Schemes to Gradient Flows, SIAM J. Numer. Anal., 56 (2018) 2895-2912.

[29] J. Shen, J. Xu, and J. Yang, The scalar auxiliary variable (SAV) approach for gradient flows, J. Comput. Phys., 353 (2018) 407-416.

[30] J. Shen, J. Xu, and J. Yang, A New Class of Efficient and Robust Energy Stable Schemes for Gradient Flows, SIAM Rev., 61 (2019) 474-506.

[31] Roger Temam, Infinite-dimensional dynamical systems in mechanics and physics, 2nd edition, Springer, 1997.

[32] V. Thomée, Galerkin finite element methods for parabolic problems, Springer-Verlag, 1997.

[33] B. Wang and $\mathrm{X}$. Wu, The formulation and analysis of energy-preserving schemes for solving high-dimensional nonlinear Klein-Gordon equations, IMA J. Numer. Anal., 39 (2019) 2016-2044.

[34] A. M. Wazwaz, New travelling wave solutions to the Boussinesq and the Klein-Gordon equations., Commun. Nonlinear Sci. Numer. Simul., 13 (2008) 889-901. 
[35] X. Wu, B. Wang, and W. Shi. Efficient energy preserving integrators for oscillatory Hamiltonian systems, J. Comput. Phys., 235 (2013) 587-605. 


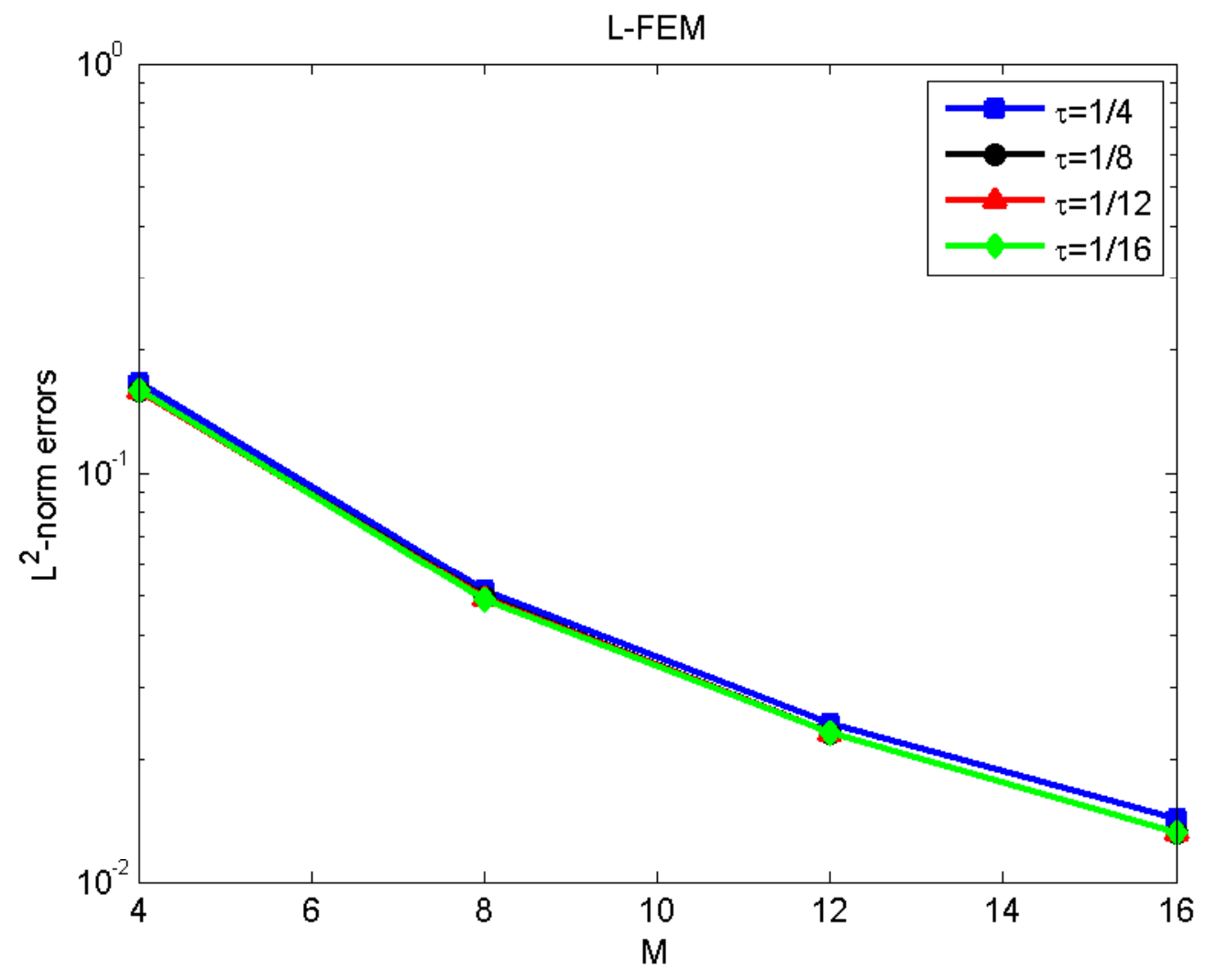




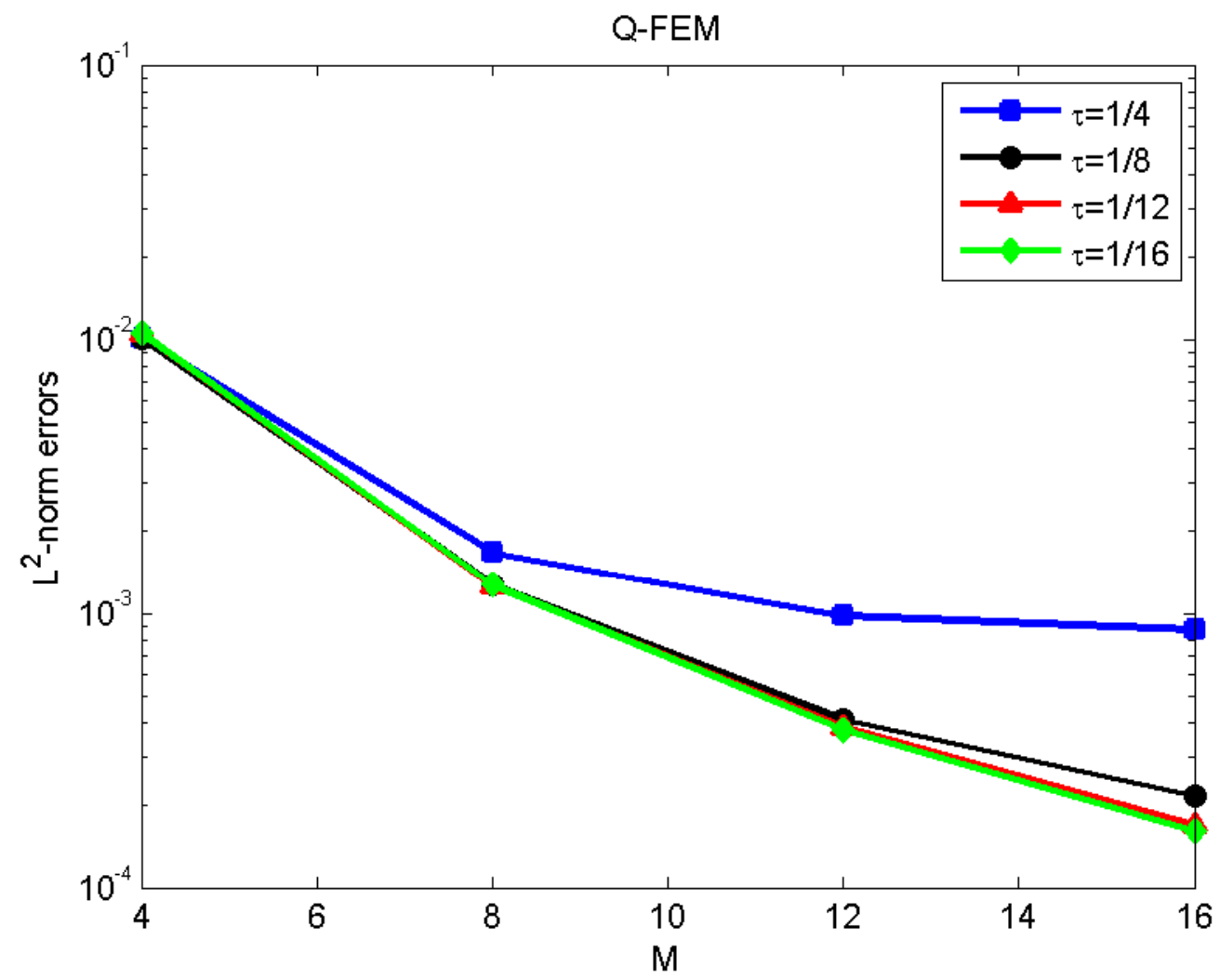




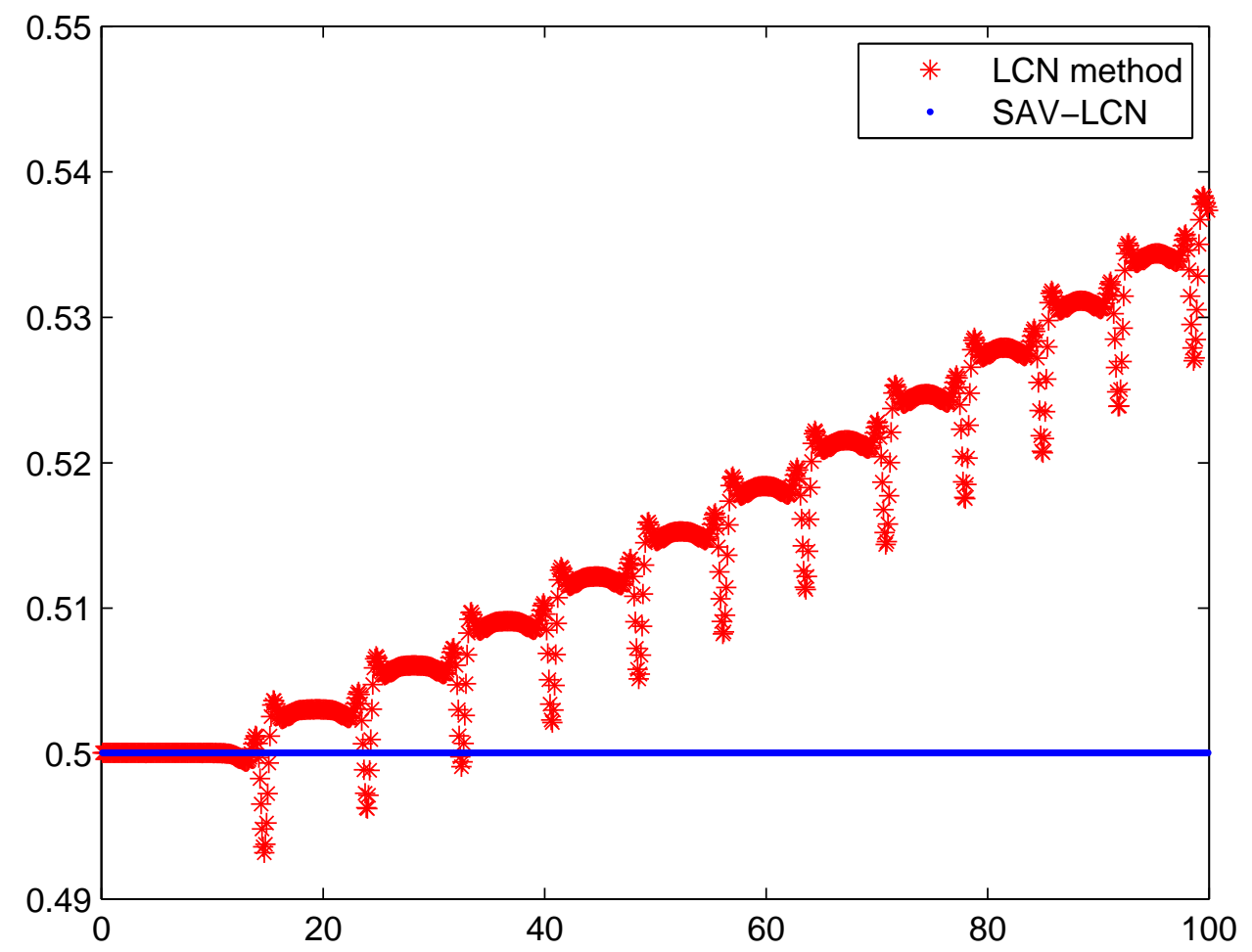

